\title{
The complement system in primary Sjögren's syndrome: the expression of certain cascade and regulatory proteins in labial salivary glands - observational study
}

\author{
Marta Legatowicz-Koprowska ${ }^{1}$, Stanisław Nitek ${ }^{2}$, Jolanta Czerwińska ${ }^{1}$ \\ ${ }^{1}$ Department of Pathology, National Institute of Geriatrics, Rheumatology and Rehabilitation, Warsaw, Poland \\ ${ }^{2}$ Department of Otolaryngology, Medical University of Warsaw, Poland
}

\begin{abstract}
Introduction: The complement cascade and regulatory proteins are involved in the pathogenesis of the Sjögren's syndrome and other autoimmune diseases. The complement activation via the alternative pathway was recognized as a major pathogenic mechanism in autoimmune conditions. The aim of this study was to assess expression of complement cascade components and regulatory proteins in minor salivary glands in patients with primary Sjögren's syndrome (pSS).

Materials and methods: The expression of $\mathrm{Clq}$ and $\mathrm{C} 5 \mathrm{~b}-9$ - membrane attack complex and regulatory proteins such as: membrane cofactor protein (MCP), decay-accelerating factor (DAF) and protectin were examined using immunochemistry method in specimens from biopsy of minor salivary glands in pSS patients. The biopsy material was obtained from 20 pSS patients, 5 patients with non-specific sialadenitis and from 5 patients with suspicion of dryness syndrome without sialadenitis confirmation.

Results: None of the examined samples showed the expression of C1q or the effector C5b-9. Membrane cofactor protein expression was lower in pSS group than in both non-specific sialadenitis and noninflamed salivary glands. The inflammatory cells in pSS samples partially expressed MCP. There were differences in the sites and intensity of membrane protectin expression exclusively on the luminal surfaces in pSS; on the luminal and, partially, antiluminal surface in non-specific inflammation, and on the entire cell surface in unaffected salivary glands. There were no DAF expression in salivary gland tissue in biopsy specimens in all studied subjects.

Conclusions: The study demonstrated the absence of complement-cascade proteins (C1q, MAC) in the salivary glands of pSS patients, which may indicated a lack of local complement activation via the classical pathway and the observed gland tissue damage being due to a mechanism other than MAC-induced cytolysis. The differences in the expression of complement regulatory proteins between pSS, non-specific sialadenitis, and normal salivary glands may indicate that alternative functions of these regulatory proteins may be of greater significance in pSS. Low MCP expression in pSS in comparison with non-specific sialadenitis and normal salivary glands, may suggest altered modulation of cell-mediated immunity in pSS. The differences in the location and intensity of protectin (CD59) expression indicates a possibility of reducing the proinflammatory effect of protectin in pSS.
\end{abstract}

Key words: primary Sjogren's syndrome, complement system proteins, membrane cofactor protein CD46, protectin CD59. 


\section{Introduction}

Sjögren's syndrome (SS) is a chronic, systemic, autoimmune disease characterized by inflammation and damage of exocrine glands, particularly salivary and lacrimal glands. The main clinical manifestation of SS is dry mouth (xerostomia) and dry eyes (xerophthalmia). This autoimmune disease may be primary (pSS); however, it is more commonly may associate other autoimmune rheumatic diseases as a secondary Sjögren's syndrome (sSS).

The current pSS classification criteria 2016 ACR/ EULAR contain the following conditions: confirmation of eye dryness by Schirmer test or/and score of ocular staining with lissamine green and fluoresceine (also Bijsterveld's method of scoring is accepted), presence of inflammatory lymphocytic infiltrates with a focus score of $\geq 1 / 4 \mathrm{~mm}^{2}$ in labial salivary glands and anti-Ro/SSA antibodies positivity [1]. Moreover, $30 \%$ of affected individuals present with polyclonal hypergammaglobulinemia, and have rheumatoid factor [2]. Less common findings (in 20-30\% of pSS patients) include detectable anticardiolipin antibodies, cryoglobulins, and low levels of complement components C3 and C4 [3].

The etiopathogenesis of pSS remains unclear, despite a number of research papers suggesting genetic, environmental, and hormonal factors potentially affecting epithelial function, autoantibody and cytokine formation, T-cell and B-cell activation, yet failing to offer a consistent presentation of the pathological process [4-7].

The characteristic anti-SSA/Ro52 autoantibodies, which are common in pSS, suggest the possibility of complement activation via the classical pathway by immune complexes (IC) formation and subsequent tissue damage. Moreover, the decrease in serum C3 and C4 levels detected in over $20 \%$ of pSS patients has not yet been explained, although it indicates the role of innate immunity in pSS pathogenesis.

\section{Complement}

The key functions of the complement system (CS) are protection against infections, removal of ICS and potentially harmful self-molecules, and modeling of the interaction between the innate and adaptive immunity $[8,9]$. The CS comprises approximately 60 mutually cooperating effector proteins, regulatory proteins, and receptors. Like many other biological systems, the CS is governed by two mechanisms: amplification and self-inhibition. Protein cascade activation leads to the formation of membrane-attack complex (MAC), which causes cell membrane perforation. The process of self-inhibition involves quenching this complement amplification by regulatory proteins to protect the body's cells from damage.
The CS can become activated via three pathways: classical, lectin, and alternative (Fig. 1). The classical pathway is induced by ICS, some viruses and bacteria, pentraxins, and cell fragments. Complement cascade activation via the lectin pathway is initiated by the binding of a mannose-binding protein to mannose residues on the pathogen surface. Lastly, the alternative pathway is activated spontaneously and continually; it predominates quantitatively over the classical pathway, and - uncontrolled - it could pose a danger to the body's cells $[8,9]$.

All three pathways converge at the stage of C 3 activation. It is the C3-convertase and its derivatives (compounds containing $(3 \mathrm{~b}$ ) that are the end-products of CS activation by most of its regulatory proteins. Membrane-bound representatives of these proteins include the decay-accelerating factor (DAF, CD55) and cofactors for the protease activity of complement factor I: membrane cofactor protein (MCP, CD46) and complement receptor 1 (CR1) [10, 11]. Soluble complement inhibitors that interact with $\mathrm{C} 3$ and its derivatives are factor $\mathrm{H}$ (for the alternative pathway) and factor I (for the classical pathway) (Fig. 1). Furthermore, protectin (CD59), anchored in the membranes of all blood cells, inhibits C9 polymerization, which protects the cell membrane from perforation. The soluble inhibitors vitronectin and clusterin exert similar effects.

There have been reports on complement regulatory protein dysfunction as an important element in the pathophysiology of autoimmune conditions [12-14].

Our search through the available literature yielded only two, partially relevant, reports on the expression of complement components and regulators in the labial salivary glands of patients with pSS $[15,16]$, despite the fact that over 20\% of those patients have low C3 and C4 levels. Further, more detailed studies on the expression of complement cascade and regulatory proteins in labial salivary glands may become the next step toward elucidating the pathogenesis of pSS, which could be useful in developing new therapies.

The aim of presented study was to assess the expression of cascade proteins in labial salivary glands such as: Clq (a marker of classical complement activation), MAC (an indicator of the complement effector function, regardless of the activation pathway), and the membrane-bound complement regulatory proteins: MCP (CD46), protectin (CD59), and DAF (CD55).

\section{Material and methods}

Our study was conducted on tissue specimens archived at the Pathology Department of National Institute of Geriatrics, Rheumatology and Rehabilitation in Warsaw between 2015 and 2017. These specimens were 


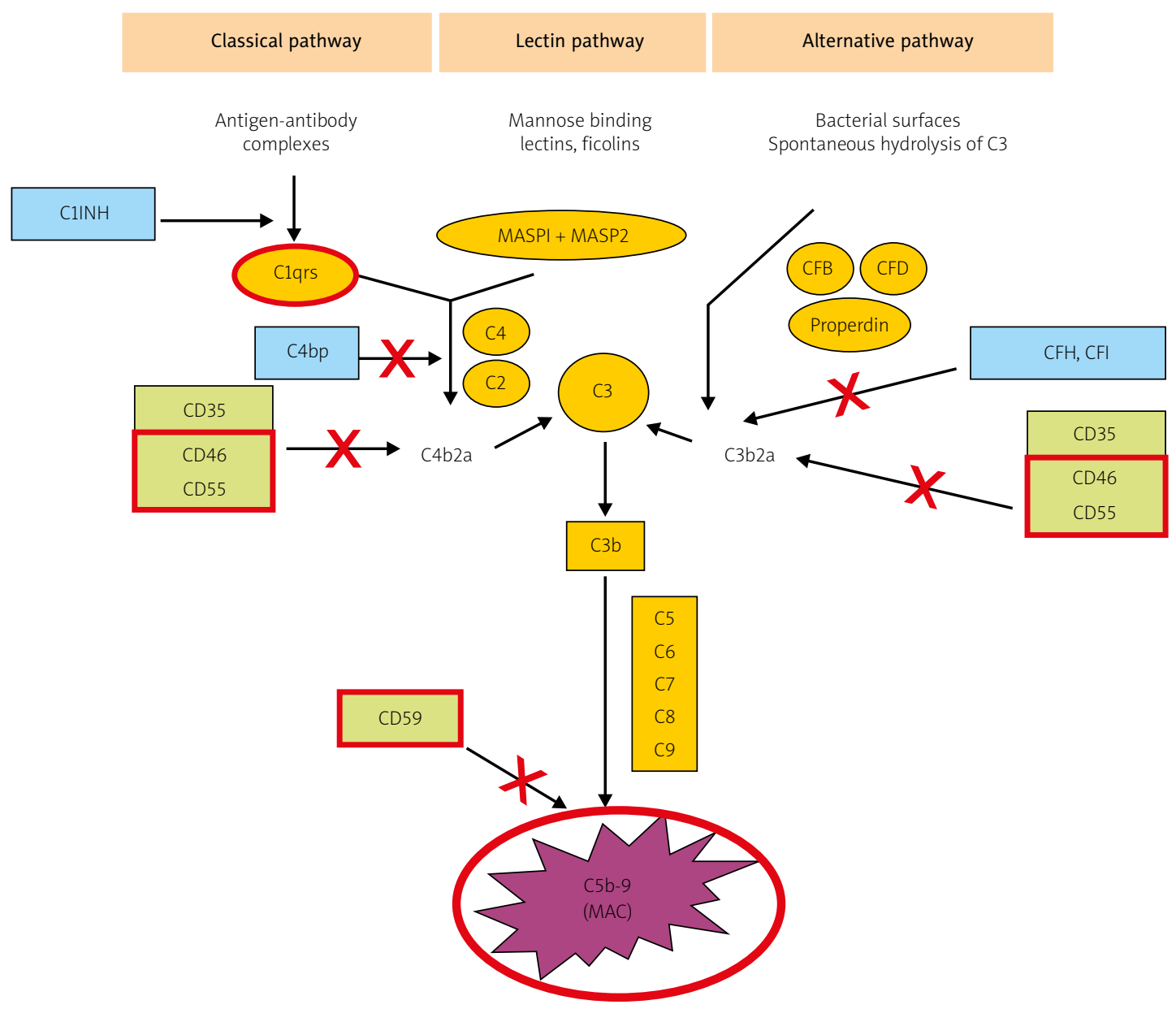

Fig. 1. The three main activation pathways of complement: the classical, mannose-binding lectin, and alternative pathways (according to Bora NS [10] - modified). The proteins under examinations in red borders.

paraffin blocks containing labial salivary gland biopsy samples collected from three patient groups (Table I):

1. Patients diagnosed with pSS based on the clinical presentation (following referral to the Pathology Department) and meeting the criterion of a focus score of $\geq 1(n=20)$.

2. Affected control group: with chronic non-specific sialadenitis $(n=5)$.

3. Control group: patients with normal salivary glands microscopic structure with no evidence of inflammation $(n=5)$.

Hematoxylin and eosin (HE)-stained specimens were examined for lymphocytic inflammatory infiltrates (focus score), secondary lymphoid follicles containing germinal centers (GC), and adenoid-tissue damage. Each sample underwent immunohistochemical (IHC) staining for $\mathrm{Clq}$ (a marker of classical complement activation), MAC (an indicator of the complement effector function, regardless of the activation pathway), and the mem-
Table I. Evaluated groups

\begin{tabular}{|lcccc|}
\hline Characteristics & $n$ & $F$ & M & Age \\
\hline $\begin{array}{l}\text { Primary Sjögren's } \\
\text { syndrome }\end{array}$ & 20 & 18 & 2 & $52(20-70)$ \\
\hline $\begin{array}{l}\text { Non-specific } \\
\text { sialadenitis }\end{array}$ & 5 & 5 & 0 & $57(41-74)$ \\
\hline $\begin{array}{l}\text { Normal salivary } \\
\text { glands }\end{array}$ & 5 & 4 & 1 & $37(17-49)$ \\
\hline
\end{tabular}

brane-bound complement regulatory proteins MCP, protectin, and DAF.

Paraffin sections ( $4 \mu \mathrm{m}$-thick) were deparaffinized in a standard way. Antigens were retrieved with Target Retrieval Solution, Low or High pH (Dako) according to the manufacturer's instructions. Endogenous peroxidase activity was blocked with $\mathrm{H}_{2} \mathrm{O}_{2}$. Abcam antibodies were diluted with Dako Antibody Diluent with Background Reducing Components to obtain optimal concentrations: 
CD46 1: 200, CD55 $1: 200$, C5b-9 $1: 1000$, CD59 $1: 100$ C1q $1: 200$. Slides were incubated with primary antibodies in a humidified chamber for 30 minutes at room temperature. Dako REALTM EnVisionTM HRP Rabbit/Mouse and DAB (Dako) were used for visualization. Cell nuclei were stained with Harris's hematoxylin. Sections were dehydrated and mounted with Histokitt (Carl Roth). Optimal antibody concentrations were determined on archived paraffin sections of lymph nodes and synovial membranes from rheumatoid arthritis patients.

These tissues also served as positive controls for $\mathrm{IHC}$ reactions. The procedures were performed without antibodies as negative controls - incubation with only Dako Antibody Diluent with Background Reducing Components.

Light microscopy was used to assess the location and classify the intensity of $\mathrm{IHC}$ reactions as negative or trace $(-)$, slightly positive $(+)$, moderately positive $(++)$, or highly positive $(+++)$. The expression of individual evaluated cell-membrane proteins was assessed in acinar (secretory) and ductal epithelial cells of salivary glands and in inflammatory cells. IHC staining for CD59 was done in such a way as to assess whether this molecule was expressed on the entire surfaces of acinar and ductal cells or on their luminal surfaces only. IHC reactions were also evaluated on endothelial surfaces and within vascular lumina (plasma).

The approval of the local ethics committee at the National Institute of Geriatrics, Rheumatology and Rehabilitation, Warsaw, Poland was obtained (decision 30.01.2020).

\section{Results}

Salivary gland biopsy specimens from patients diagnosed with pSS yielded focus scores ranging from 1 to 5 . Glandular structure damage was observed in 11 out of the 20 evaluated cases, whereas 3 specimens contained secondary lymphoid follicles (Table II). HE staining in the 5 evaluated cases of chronic non-specific sialadenitis (affected controls) showed dispersed lymphocytes. The 5 non-affected controls showed unremarkable microscopic structure of the salivary glands, with no inflammatory infiltrates.

All cases of anti-Clq IHC staining of gland structures in the three study groups were negative (Tables II, III, IV), with only slightly positive $(+)$ reactions observed in the plasma (in some vascular lumina), which proved the correct execution of staining protocols.

Likewise, no MAC (C5b-9) expression was detected in gland tissue from either pSS or non-specific sialadenitis patients, with only slightly positive reactions observed in the plasma (Tables II, III). Normal salivary glands stained with anti-C5b-9 showed negative reactions (Table IV).

Anti-MCP (CD46) IHC staining produced varied reactions. Fifty percent of the salivary glands with a focus score of $\geq 1$ showed no MCP expression either on acinar or ductal cell membranes. The remaining glands showed a slightly positive (+) or inconsistent reaction (10-20\% of acinar cells and few ducts). More intense reactions (from + to +++ ) were observed on the cell membranes of some infiltrate-forming lymphocytes (Fig. 2A). There were no differences between biopsy samples with and without glandular tissue damage or those with or without secondary lymphoid follicles.

Non-specific sialadenitis specimens showed markedly positive $(++/+++)$ reactions with anti-MCP antibodies on the surface of ductal epithelium (Fig. 2B). Acinar and inflammatory cell membranes produced focal, less pronounced $(+)$ reactions.

Non-affected control salivary gland specimens produced a homogeneously pronounced positive reaction $(+++)$ with anti-MCP on nearly all acinar and ductal cell membranes (Fig. 2C).

Protectin (CD59) expression in pSS glands varied in intensity from $(+)$ to $(+++)$ and was present only on the luminal surface of acinar and ductal cells (Fig. 2D). Non-specific sialadenitis specimens showed less intense luminal-surface reactions $(+) /(++)$, with positive $(+) /(++)$ reactions observed on the entire surface of acinar cells (Fig. 2E). Non-affected control salivary glands showed inconsistent $\mathrm{IHC}$ reactions; with predominantly highly positive reactions on the entire surfaces of acinar and ductal epithelial cells (Fig. 2F).

Slightly positive IHC reactions with anti-DAF (CD55) were present in some pSS and non-specific sialadenitis specimens, though only on paraglandular connective-tissue fibers. Normal salivary glands showed no DAF expression.

Tables II, III, and IV illustrate IHC reaction results.

\section{Discussion}

Labial salivary glands constitute adequate specimens for the assessment of the type and intensity (focus score) of chronic inflammation in the diagnostics and monitoring of pSS. Inflammatory cell infiltrates may disturb glandular function in several mechanisms (by tissue damaging cytotoxic cells, the secretion of cytokines that activate interferon-associated pathways, or local autoantibody production) [5]. Our study on the expression of bioactive molecules (i.e. complement components and regulatory proteins) may be an important step towards elucidating these mechanisms. 


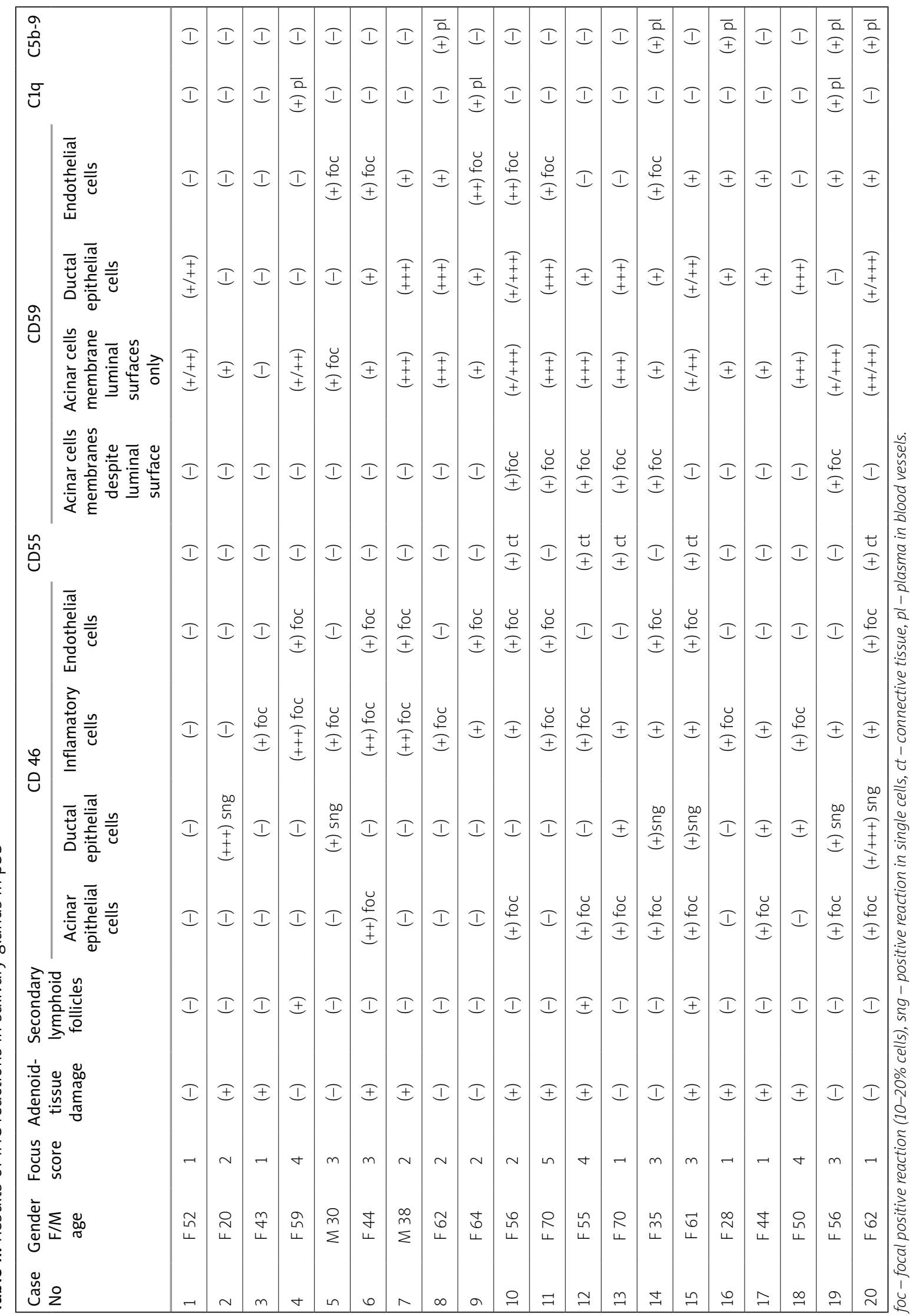



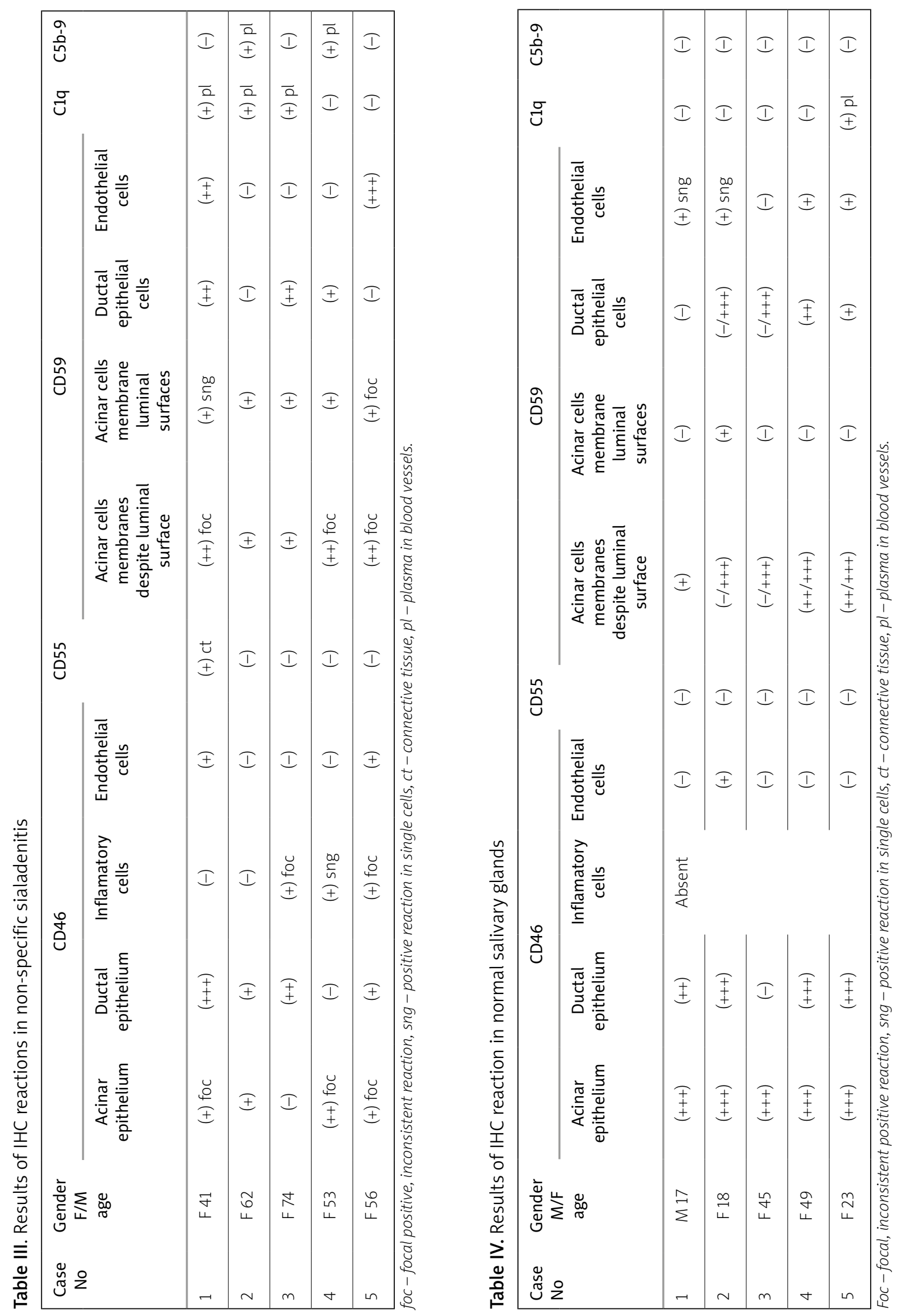

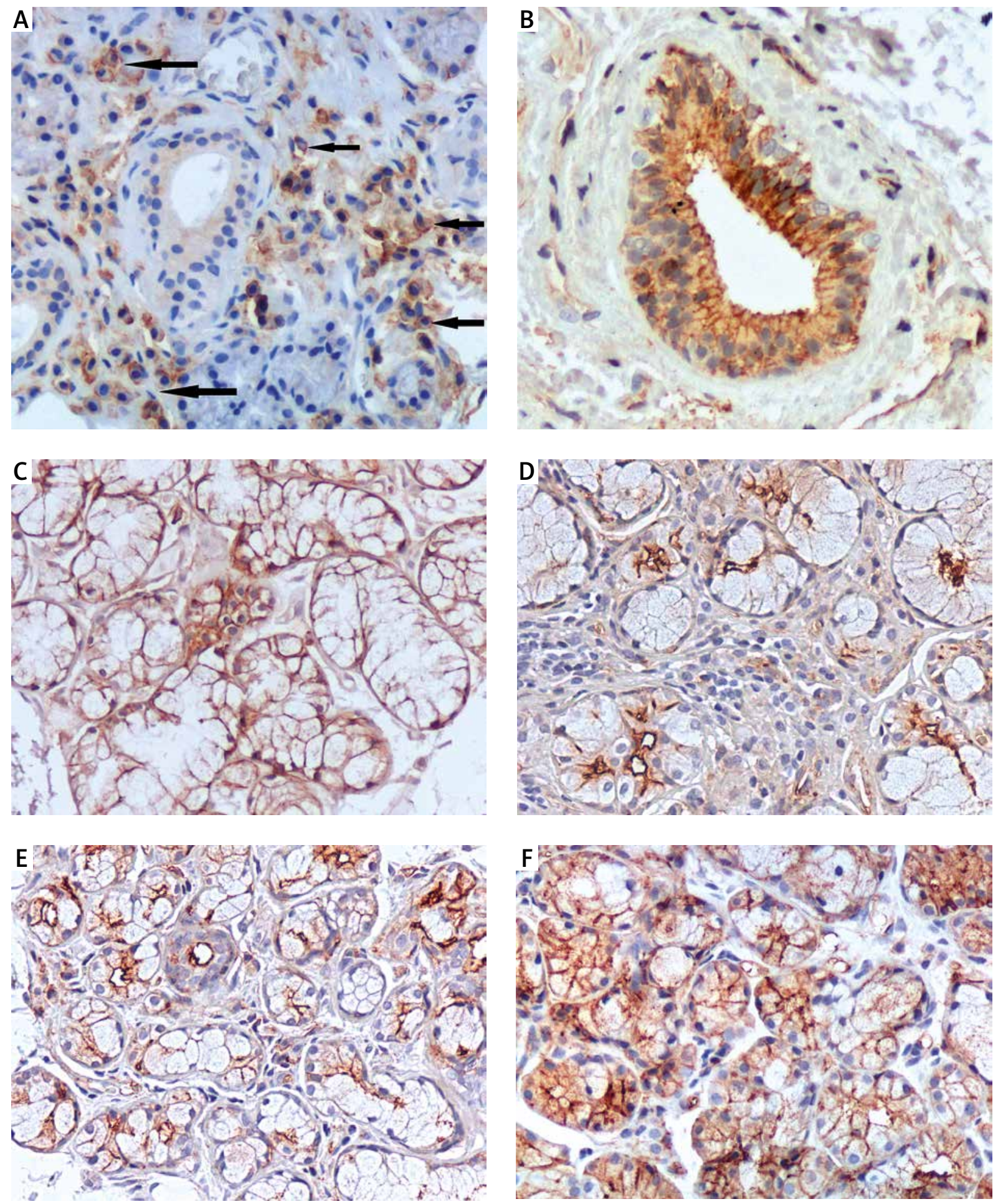

Fig. 2. IHC staining for CD46 (A-C). pSS - intensive reaction of lymphocytes - arrows (A), non-specific sialadenitis: markedly positive (+++) reactions on the surface of ductal epithelium (B), normal salivary glands: homogeneously positive reaction (+++) with anti-MCP on nearly all acinar cell membranes (C). IHC staining for CD59 (D-F). pSS - CD59 homogeneously pronounced positive expression only on luminal surface of acinar and ductal cells (D), non-specific sialadenitis: luminal-surface reactions $(+) /(++)$, with positive $(+) /(++)$ reactions observed on the entire surface of acinar cells $(E)$, normal salivary glands: predominantly highly positive reactions on the entire surfaces of acinar and ductal epithelial cells (F). All pictures magnification $\times 200$. 
There have been a relatively large number of reports on low serum C3 and C4 levels in pSS patients and the predictive value of this finding for lymphoma development $[2,3]$. However, the distribution of complement components and regulators in salivary glands has been addressed scantily and incompletely $[15,16]$.

Our study showed no expression of either C1q or MAC in the labial salivary glands classified as affected by SS. This suggests that the autoimmune process of pSS involves no local complement activation via the classical (no C1q expression), alternative, or lectin (no MAC expression) pathways. The lack of local classical-pathway activation - despite evidence of peripheral complement component depletion (low serum C3 and C4 levels) - may indicate that the formation of ICs in salivary glands is not an important part of the inflammatory process and the observed tissue damage results from a different mechanism than MAC-induced cytolysis. Interestingly, a Chinese study [16] demonstrated positive Clq expression via IHC staining in labial salivary glands of 71 patients with pSS The discrepancy between those and our findings may be due to their use of Zhongjin Jinqiao Biotechnology Co. (Beijing China) rabbit polyclonal antibodies and our use of Abcam mouse anti-Clq monoclonal antibodies.

The expression of complement regulatory proteins MCP (CD46), DAF (CD55), protectin (CD59) on acinar cell membranes in normal salivary glands was already demonstrated in the early 1990s [17-19].

Membrane-attack complex (CD46) is a transmembrane protein present on all nucleated cells. Its main role as a cofactor for factor I in C3b and C4b cleavage (which halts component-cascade activation) is to protect autologous cells from damage by MAC. MCP is also a T-cell co-stimulator, inducing T-cell (CD4+) differentiation into Treg, which secrete anti-inflammatory cytokines (e.g. IL-10) and thus suppress immune response and prevent autoimmunization [20, 21].

IHC staining of salivary glands showed no expression of CD46 in acinar or ductal cells in $50 \%$ of pSS patients, with slight $(+)$ or inconsistent responses in the remaining half. Conversely, lymphocytes showed locally high expression of CD46 - from (+) to $(+++)$ - particularly on the periphery of inflammatory foci. Study results reported by Cuida at al. [15] were somewhat different, with MCP expression observed on the luminal surfaces of acinar and ductal epithelium. However, the study sample size should not be overlooked, with 20 cases evaluated in our study and 5 in cited study. Nonetheless, our findings are consistent with those reported by authors regarding CD46 expression on inflammatory cell membranes in pSS. This leaves room for further research to determine the phenotype of inflammatory cells expressing CD46, which might show whether MCP expression indeed correlates with T-cells obtaining the Treg anti-inflammatory phenotype.

It is difficult to explain the highly positive $\mathrm{IHC}$ reaction with anti-CD46 in non-specific sialadenitis being limited to ductal epithelial cells (Fig. 2B), with a less pronounced reaction observed on acinar and inflammatory cells. Likewise inexplicable is the fact that CD46 expression in normal salivary glands was different, with a homogeneous, highly positive reaction on acinar and ductal epithelial cells (Fig. 2C). Is it only such "expansive" CD46 expression that protects salivary gland cells from autoimmunity-induced destruction? Questions also arise about possible complement modulators. Answering these questions calls for employing a different study methodology (evaluating gene transcription and protein incorporation into cell membranes).

Protectin, or membrane inhibitor of reactive lysis (MIRL, CD59), is a glycosylphosphatidylinositol (GPI) - anchored membrane-bound glycoprotein, which is a structural component of peripheral blood cells (leukocytes and erythrocytes). Protectin is expressed on the endothelial surface and epithelia of exocrine-glands (including salivary glands) [18]. It inhibits the formation of MAC (C5b-9) by binding to C8 and C9 and preventing the incorporation of multiple C9 molecules into MAC. Moreover, protectin plays a role in signal transduction for T-cell activation and thus modulates cell-mediated immunity [22-25]. Another, recently suggested role of CD59 was that its activation on target-cell membranes may potentiate NK-cell-mediated cytotoxicity [24]. Additionally, CD59 expressed on NK-cells may enhance their cytolytic activity [25].

Similar to the Cuida et al. research [15], our study showed CD59 expression on luminal surfaces of acinar and ductal epithelial cells in pSS (Fig. 2D). We observed no differences in CD59 expression in salivary glands with and without microscopic evidence of glandular tissue damage. Therefore, this finding provided no explanation as to how the presence of CD59 on gland cells may affect the extent of their damage. Nonetheless, the noticeable differences in CD59 expression in the salivary glands in pSS and those in non-specific sialadenitis with weaker $\mathrm{IHC}$ reaction intensity in the latter (Fig. 2E) - demonstrated a dissimilar character of inflammatory processes in these two conditions. IHC staining showed no protectin on inflammatory-cell membranes.

One interesting finding was that normal salivary glands showed marked (++/+++) CD59 expression all over acinar cells, not only on their luminal surface (Fig. 2F). This distinction was not shown in Cuida et al. [15] work. Although CD59 expression have been long shown to be affected by cytokines $[14,26]$, its regulatory mechanisms are not fully understood. CD59 may reach the 
cell membrane via exocytosis [27] and be subsequently freed from its GPI bond via specific phospholipase C or proteolytic enzymes [28]. Possibly, during the course of sialadenitis, tissue cytokines alter CD59 expression on acinar cell membrane, whereupon it is left only on the luminal surface (which is not in direct contact with surrounding tissues). This theory requires further studies.

Interestingly, in rheumatoid arthritis (RA) CD59 expression was shown to be absent on synovial cells and reduced on interstitial cells and the endothelial cells of synovial membrane blood vessels [14]. Moreover, studies on an antigen-induced arthritis (AIA) model in CD59deficient mice demonstrated an increased number of MACs in the synovial membrane - and, consequently, greater tissue damage compared with the control groupwith subsequent improvement following CD59-deficiency correction [29]. It turns out that one of the mechanisms of the anti-inflammatory effect of statins observed in RA mice may be an increased CD59 expression in tissues, which results in reduced complement activation [30]. A similar anti-inflammatory effect was observed in about a dozen clinical trials in RA patients [31].

Decay-accelerating factor (CD55) is a globular glycoprotein anchored to the cell membranes of blood cells, cells in a direct contact with plasma, and epithelial cells lining body cavities [19]. Decay-accelerating factor inhibits the formation of C3 and C5 convertases, thus limiting complement cascade activation [32]. It also acts as a modulator reducing the T-cell-mediated response [33]. As a CD97 ligand, DAF (together with CD97) induces T-cell and B-cell proliferation [32].

Surprisingly, our study showed no CD55 expression either in normal salivary glands or those affected by non-specific sialadenitis or pSS. Trace CD55 expression was only detected in interstitial salivary gland tissue, which was consistent with previous Cuida's et al. [15] findings. In models of various inflammatory diseases, including autoimmune ones, the lack of CD55 has been associated with a greater severity of clinical manifestations [34]. However, Hoek et al. [35] demonstrated that a lack of CD55 in a murine RA model was associated with a reduced severity of synovitis. These seemingly contradictory findings may be explained when accounting for varied CD55 activity depending on the surrounding cells [32].

The most recent studies suggest that CD55 may be a positive regulator of tumor formation and malaria, while being a negative regulator of autoimmune and demyelinating conditions, paroxysmal nocturnal hemoglobinuria, and protein losing enteropathy [32].

\section{Study limitations}

This study needs further processing of results in relationship with clinical data. The relatively small study group and control group limited the value of a statistic analysis, what positioned the work as an observational study. An additional limitation was that only one pathologist assessed the location and intensity of $\mathrm{IHC}$ reactions.

\section{Conclusions}

Our study demonstrated the absence of complement-cascade proteins (C1q, MAC) in the salivary glands of pSS patients, which indicated a lack of local complement activation via the classical pathway and the observed gland tissue damage being due to a mechanism other than MAC-induced cytolysis.

The observed differences in the expression of complement regulatory proteins in pSS, non-specific sialadenitis, and normal salivary glands in the absence of complement cascade activation may indicate that alternative functions of these regulatory proteins (e.g. modulation of cell-mediated response) may be of greater significance in pSS.

Low MCP expression in the salivary glands of patients with pSS, in comparison with that in the salivary glands in non-specific sialadenitis and that in normal salivary glands, may suggest altered modulation of cell-mediated immunity in the autoimmune process of pSS. Moreover, the differences in the location and intensity of protectin (CD59) expression in these three types of salivary gland samples indicates a possibility of reducing the proinflammatory effect of protectin in pSS.

\section{Acknowledgements}

We thank professor Aleksander Wasiutynski PhD for the conception of the research. We also thank Mrs. Malgorzata Wojcik and Mr. Adam Wojcik for the technical assistance.

\section{Funding}

This study was supported by the National Institute of Geriatrics, Rheumatology and Rehabilitation, Warsaw, Poland.

The authors declare no conflict of interest.

\section{References}

1. Shiboski CH, Shiboski SC, Seror R, et al. Criteria 2016 ACR-EULAR Classification Criteria for primary Sjögren's Syndrome: A Consensus and Data-Driven Methodology Involving Three International Patient Cohorts. Arthritis Rheumatol 2017; 69: 35-45, DOI: 10.1002/art.39859.

2. Carubbi F, Alunno A, Cipriani P, et al. A retrospective, multicenter study evaluating the prognostic value of minor salivary gland histology in a large cohort of patients with pri- 
mary Sjögren's syndrome. Lupus 2015; 24: 315-320, DOI: 10.1177/0961203314554251.

3. Ramos-Casals M, Brito-Zerón P, Yagüe J, et al. Hypocomplementaemia as an immunological marker of morbidity and mortality in patients with primary Sjögren's syndrome Rheumatology (Oxford) 2005; 44: 89-94, DOI: 10.1093/rheumatology/keh407.

4. Peri Y, Agmon-Levin N, Theodor E, Shoenfeld Y. Sjögren's syndrome, the old and the new. Best Pract Res Clin Rheum 2012; 26: 105-117, DOI: 10.1016/j.berh.2012.01.012.

5. Nair JJ, Singh TP. Sjogren syndrome: Review of the aetiology, pathophysiology \& potential therapeutic interventions. J Clin Exp Dent 2017; 9: e584-e589.

6. Cruz-Tapias P, Rojas-Villarraga A, Maier-Moore S, Anaya JM HLA and Sjögren's syndrome susceptibility. A meta-analysis of worldwide studies. Autoimmun Rev 2012; 11: 281-287, DOI: 10.1016/j.autrev.2011.10.002.

7. Voulgarelis M, Tzioufas AG. Pathogenetic mechanisms in the initiation and perpetuation of Sjogren's syndrome. Nat Rev Rheumatol 2010; 6: 529-537, DOI: 10.1038/nrrheum.2010.118.

8. Le Friec G, Kemper C. Complement: coming full circle. Arch Immunol Ther Exp (Warsz) 2009; 57: 393-407, DOI: 10.1007/ s00005-009-0047-4.

9. Walport MJ. Complement. First of Two Parts. N Engl J Med 2001; 344: 1058-1066, DOI: 10.1056/NEJM200104053441406.

10. Bora NS, Matta B, Lyzogubov VV, Bora PS. Relationship between the complement system, risk factors and prediction models in age-related macular degeneration. Mol Immuno 2015; 63: 176-183, DOI: 10.1016/j.molimm.2014.07.012.

11. Kim DD, Song W-C. Membrane complement regulatory proteins. Clin Immunol 2006; 118: 127-136, DOI: 10.1016/j.clim.2005. 10.014 .

12. Chen M, Daha MR, Kallenberg CGM. The complement system in systemic autoimmune disease. J Autoimmun 2010; 34 J276-J286, DOI: 10.1016/j.jaut.2009.11.014.

13. Holers VM, Thurman JM. The alternative pathway of complement in disease: opportunities for therapeutic targeting. Mol Immunol 2004; 41: 147-152, DOI: 10.1016/j.molimm.2004.03.012

14. Konttinen YT, Ceponis A, Meri S, et al Complement in acute and chronic arthritides: assessment of C3c, C9, and protectin (CD59) in synovial membrane. Ann Rheum Dis 1996; 55: 888-894.

15. Cuida M, Legrer DW, Eidsheim M, Jonsson R. Complement regulatory proteins in the salivary glands and saliva of Sjögren's syndrome patients and healthy subjects. Clin Exp Rheumato 1997; 15: 615-623

16. Sun W, Zhang N, Zhang Y, et al. Immunophenotypes and clinical features of lymphocytes in the labial gland of primary Sjogren's syndrome patients. J Clin Lab Anal 2018; 32: e22585, DOI: $10.1002 /$ jcla.22585.

17. Meri S, Waldmann H, Lachmann PJ. Distribution of protectin (CD59), a complement membrane attack inhibitor in normal human tissues. Lab Invest 1991; 65: 532-537.

18. Johnstone W, Loveland BE, McKenzie IFC. Identification and quantification of complement regulator CD46 on normal human tissues. Immunology 1993; 79: 341-347.

19. Medof ME, Walter El, Rutgers JL, et al. Identification of the complement decay accelerating factor (DAF) on epithelium and glandular cells and in body fluids. J Exp Med 1987; 165 848-864, DOI: 10.1084/jem.165.3.848.

20. Carroll MC. The complement system in regulation of adaptive immunity. Nat Immunol 2004; 5: 981-986.
21. Kemper C, Chan AC, Jonathan MG, et al. Activation of human CD4 cells with CD3 and CD46 induces a T regulatory cell 1 phenotype. Nature 2003; 421: 388-392, DOI: 10.1038/nature01315.

22. Korty PE, Brando C, Shevach EM. CD59 functions as a signal transducing molecule for human T cell activation. J Immunol 1991; 146: 4092-4098.

23. Kimberley FC, Sivasankar B, Morgan BP. Alternative roles for CD59. Mol Immunol 2007; 44: 73-81, DOI: 10.1016/j.molimm. 2006.06.019.

24. Marcenaro E, Augugliaro R, Falco M, et al. CD59 is physically and functionally associated with natural cytotoxicity receptors and activates human NK cell-mediated cytotoxicity. Eur J Immunol 2003; 33: 3367-3376, DOI: 10.1002/eji.200324425.

25. Omidvar N, Wang PEC, Brennan P, et al. Expression of glycosylphosphatidylinositol-anchored CD59 on target cells enhances human NK cell-mediated cytotoxicity. J Immunol 2006; 176: 2915-2923, DOI: 10.4049/jimmunol.176.5.2915.

26. Bjørge L, Jensen TS, Ulvestad $E$ et al. The influence of tumour necrosis factor- $\alpha$, interleukin- $1 \beta$ and interferon- $\gamma$ on the expression and function of the complement regulatory protein CD59 on the human colonic adenocarcinoma cell line HT29. Scand J Immunol 1995; 41: 350-356, DOI: 10.1111/j.1365-3083. 1995.tb03578.x.

27. Väkevä A, Laurila P, Meri S. Loss of expression of protectin (CD59) is associated with complement membrane attack complex deposition in myocardial infarction. Lab Invest 1992; 67: 608-616.

28. Holguin MH, Wilcox LA, Bernshaw NJ, et al. Erythrocyte membrane inhibitor of reactive lysis: effects of phosphatidylinositol-specific phospholipase $C$ on the isolated and cell associated protein. Blood 1990; 75: 284-289

29. Williams AS, Mizuno M, Richards PJ, et al. Deletion of the gene encoding CD59a in mice increases disease severity in a murine model of rheumatoid arthritis. Arthritis Rheum 2004; 50: 3035-3044, DOI: 10.1002/art.20478.

30. Kinderlerer AR, Steinberg R, Johns M, et al. Statin-induced expression of CD59 on vascular endothelium in hypoxia: a potential mechanism for the anti-inflammatory actions of statins in rheumatoid arthritis. Arthritis Res Ther 2006; 8: R130, DOI: 10.1186/ar2019.

31. Li GM, Zhao J, Li B, et al. The anti-inflammatory effects of statins on patients with rheumatoid arthritis: A systemic review and meta-analysis of 15 randomized controlled trials. Autoimmun Rev 2018;17: 215-225, DOI: 10.1016/j.autrev.2017.10.013.

32. Dho SH, Lim JC, Kim LK. Beyond the Role of CD55 as a Complement Component. Immune Netw 2018; 18: e11, DOI: 10.4110/ in.2018.18.e11.

33. Capasso M, Durrant LG, Stacey M, et al. Costimulation via CD55 on human CD4+ T cells mediated by CD97. J Immunol 2006;177: 1070-1077, DOI: 10.4049/jimmunol.177.2.1070.

34. Miwa T, Sun X, Ohta R, et al. Characterization of glycosylphosphatidylinositol-anchored decay accelerating factor (GPI-DAF) and transmembrane DAF gene expression in wild-type and GPI-DAF gene knockout mice using polyclonal and monoclonal antibodies with dual or single specificity. Immunology 2001;104: 207-214, DOI: 10.1046/j.1365-2567.2001.01280.x.

35. Hoek RM, de Launay D, Kop EN, et al. Deletion of either CD55 or CD97 ameliorates arthritis in mouse models. Arthritis Rheum 2010; 62: 1036-1042, DOI: 10.1002/art.27347. 